\title{
A STRONG STIELTJES MOMENT PROBLEM
}

\author{
BY
}

\author{
WILLIAM B. JONES, W. J. THRON AND HAAKON WAADELAND ${ }^{1}$
}

\begin{abstract}
This paper is concerned with double sequences of complex numbers $C=\left\{c_{n}\right\}_{-\infty}^{\infty}$ and with formal Laurent series $L_{0}(C)=\Sigma_{1}^{\infty}-c_{-m} z^{m}$ and $L_{\infty}(C)=$ $\Sigma_{0}^{\infty} c_{m} z^{-m}$ generated by them. We investigate the following related problems: (1) Does there exist a holomorphic function having $L_{0}(C)$ and $L_{\infty}(C)$ as asymptotic expansions at $z=0$ and $z=\infty$, respectively? (2) Does there exist a real-valued bounded, monotonically increasing function $\psi(t)$ with infinitely many points of increase on $[0, \infty)$ such that, for every integer $n, c_{n}=\int_{0}^{\infty}(-t)^{n} d \psi(t)$ ? The latter problem is called the strong Stieltjes moment problem. We also consider a modified moment problem in which the function $\psi(t)$ has at most a finite number of points of increase. Our approach is made through the study of a special class of continued fractions (called positive $T$-fractions) which correspond to $L_{0}(C)$ at $z=0$ and $L_{\infty}(C)$ at $z=\infty$. Necessary and sufficient conditions are given for the existence of these corresponding continued fractions. It is further shown that the even and odd parts of these continued fractions always converge to holomorphic functions which have $L_{0}(C)$ and $L_{\infty}(C)$ as asymptotic expansions. Moreover, these holomorphic functions are shown to be represented by Stieltjes integral transforms whose distributions $\psi^{(0)}(t)$ and $\psi^{(1)}(t)$ solve the strong Stieltjes moment problem. Necessary and sufficient conditions are given for the existence of a solution to the strong Stieltjes moment problem. This moment problem is shown to have a unique solution if and only if the related continued fraction is convergent. Finally it is shown that the modified moment problem has a unique solution if and only if there exists a terminating positive $T$-fraction that corresponds to both $L_{0}(C)$ and $L_{\infty}(C)$. References are given to other moment problems and to investigations in which negative, as well as positive, moments have been used.
\end{abstract}

1. Introduction. In this paper we are concerned with double sequences of complex numbers

$$
C=\left\{c_{n}\right\}_{-\infty}^{\infty}, \quad c_{n} \in \mathbf{C},
$$

and with formal Laurent series (fLs) generated by them as follows

$$
L_{0}(C)=\sum_{m=1}^{\infty}-c_{-m} z^{m}, \quad L_{\infty}(C)=\sum_{m=0}^{\infty} c_{m} z^{-m}
$$

We seek to determine functions $G(z)$, holomorphic for $z$ in some open region $D$, having 0 and $\infty$ as boundary points, which have $L_{0}(C)$ and $L_{\infty}(C)$ as asymptotic expansions, with respect to $D$, at $z=0$ and $z=\infty$, respectively.

Received by the editors April 24, 1979.

AMS (MOS) subject classifications (1970). Primary 30A90, 30A84, 30A86; Secondary 30A22, 30 A82.

Key words and phrases. Moment problems, asymptotic expansions, continued fractions, integral representations.

${ }^{1}$ Research supported in part by the United States National Science Foundation under Grant No. MCS 78-02152 and by the Norwegian Research Council for Science and Humanities. 
We recall (see, for example, the recent treatment by Henrici [2, Chapter 11]) that a series $\sum_{m=0}^{\infty} d_{m} z^{-m}$, is called an asymptotic expansion of $f(z)$ at $z=\infty$, with respect to a region $S$ which has $\infty$ as a boundary point, if there exist sequences of positive numbers $\left\{\eta_{n}\right\}$ and $\left\{\rho_{n}\right\}$ such that, for each $n=0,1,2, \ldots$,

$$
\left|f(z)-\sum_{m=0}^{n} d_{m} z^{-m}\right| \leqslant \eta_{n}|z|^{-n-1} \text { for }|z|>\rho_{n}, z \in S .
$$

Similarly, $\sum_{m=0}^{\infty} k_{m} z^{m}$ is called an asymptotic expansion of $g(z)$ at $z=0$, with respect to a region $U$ which has 0 on its boundary, if there exist sequences of positive numbers $\left\{\sigma_{n}\right\}$ and $\left\{\delta_{n}\right\}$ such that, for each $n=0,1,2, \ldots$,

$$
\left|g(z)-\sum_{m=0}^{n} k_{m} z^{m}\right| \leqslant \sigma_{n}|z|^{n+1} \text { for }|z|<\delta_{n}, z \in U .
$$

One way of obtaining solutions to the above problem is to ask under what conditions on the double sequence $C$ is it possible to find a general $T$-fraction,

$$
\frac{F_{1} z}{1+G_{1} z}+\frac{F_{2} z}{1+G_{2} z}+\frac{F_{3} z}{1+G_{3} z}+\cdots,
$$

satisfying $F_{n}>0, G_{n}>0$ for all $n \geqslant 1$, which corresponds to $L_{0}(C)$ at $z=0$ and to $L_{\infty}(C)$ at $z=\infty$. The definition of correspondence will be given in $\$ 2$. Unless otherwise stated, continued fractions in this article will be nonterminating.

Hereafter, general $T$-fractions (1.4) satisfying $F_{n}>0, G_{n}>0$ for all $n>1$ shall be called positive $T$-fractions.

The notation $H_{k}^{(n)}(C)$ will be used for Hankel determinants as follows:

$$
H_{k}^{(n)}(C)=\left|\begin{array}{cccc}
c_{n} & c_{n+1} & \cdots & c_{n+k-1} \\
c_{n+1} & c_{n+2} & \cdots & c_{n+k} \\
\vdots & \vdots & & \vdots \\
c_{n+k-1} & c_{n+k} & \cdots & c_{n+2 k-2}
\end{array}\right|,
$$

The conditions on the double sequence $C$ for there to exist a positive $T$-fraction corresponding to $L_{0}(C)$ and $L_{\infty}(C)$, respectively, will be shown to be

$$
H_{n+1}^{(-n)}(C)>0, n \geqslant 0 ; H_{2 n}^{(-2 n)}(C)>0, H_{2 n-1}^{(-2 n+1)}(C)<0, n \geqslant 1 .
$$

Positive $T$-fractions are shown to have integral representations of the form

$$
G(z)=\int_{0}^{\infty} \frac{z d \psi(t)}{z+t}, \quad z \in R, \quad \psi \in \Psi .
$$

Here

$$
R=[z:|\arg z|<\pi],
$$

and $\Psi$ is the set of all real-valued monotone nondecreasing functions $\psi(t)$ defined on $0 \leqslant t<\infty$, with $\psi(0)=0$ and $\lim _{t \rightarrow \infty} \psi(t)<\infty$. $\Psi$ is further subdivided into the set $\Psi_{F}$ of those functions which have only a finite number of points of increase and the set $\Psi_{\infty}$ of those functions $\psi$ having an infinite number of points of increase. 
In addition to $R$ it is convenient to introduce the regions

$$
R_{\alpha}=[z:|\arg z|<\alpha], \quad 0<\alpha<\pi .
$$

The function $\psi$ occurring in (1.6) will be in $\Psi_{F}$ iff the positive $T$-fraction is terminating and in $\Psi_{\infty}$ if the $T$-fraction is nonterminating. Further, the function $\psi$ in (1.6) satisfies the conditions

$$
c_{n}=\int_{0}^{\infty}(-t)^{n} d \psi(t), \quad n=0, \pm 1, \pm 2, \ldots,
$$

and is thus a solution of a moment problem for the double sequence $C$. By a strong Stieltjes moment problem we shall mean the following: For a given double sequence $C=\left\{c_{n}\right\}_{-\infty}^{\infty}$, does there exist a $\psi \in \Psi_{\infty}$ satisfying (1.8)? There also is a moment problem for $\psi \in \Psi_{F}$. This will be called a modified moment problem.

The functions $G(z)$ defined in (1.6) have the series $L_{0}(C)$ and $L_{\infty}(C)$ as asymptotic expansions, with respect to $R_{\alpha} \subset R, 0<\alpha<\pi$, at $z=0$ and $z=\infty$, respectively.

A natural question to ask is the following: If a general $T$-fraction converges to a function $f(z)$, holomorphic in a region $D$, having 0 and $\infty$ on its boundary, are the fLs, to which the general $T$-fraction corresponds, asymptotic expansions of $f(z)$ with respect to some region $D^{\prime} \subset D$ ? Surprisingly the only general class of cases known so far in which the question has an affirmative answer is that of the positive $T$-fractions.

Other questions relating to a doubly infinite sequence of moments have been previously considered by Covindarajula [1], Kabe [7], Mendenhall and Lehman [10] and Thomas [14].

The contents of this paper are as follows. In \$2, necessary and sufficient conditions for the existence of a general $T$-fraction corresponding to two given fLs are obtained. $\S 3$ is devoted to a study of positive $T$-fractions. We characterize those double sequences $C$ for which there is a positive $T$-fraction corresponding to $L_{0}(C)$ and $L_{\infty}(C)$. The convergence behavior of positive $T$-fractions is then investigated and it is shown that the odd and even parts of positive $T$-fractions always have integral representations. In $\$ 4$ we establish that (1.8) holds for the given double sequence $C$ and the functions $\psi^{(\sigma)}$ obtained from the integral representations of the positive $T$-fraction corresponding to $L_{0}(C)$ and $L_{\infty}(C)$. It is also proved that the functions $G^{(\sigma)}(z)$, to which the odd and even parts of the positive $T$-fraction converge, have $L_{0}(C)$ and $L_{\infty}(C)$ as asymptotic expansions. $\S 5$ is concerned with terminating positive $T$-fractions and a solution of the modified moment problem. In $§ 6$, necessary and sufficient conditions for the solvability of the strong Stieltjes moment problem, as well as for the uniqueness of the solution, are given.

We conclude this introduction by summarizing a few elementary facts about continued fractions that will subsequently be used. A continued fraction is an ordered pair $\left\langle\left\langle\left\{a_{n}\right\},\left\{b_{n}\right\}\right\rangle,\left\{f_{n}\right\}\right\rangle$, where $a_{1}, a_{2}, \ldots$ and $b_{0}, b_{1}, b_{2}, \ldots$ are complex numbers with $a_{n} \neq 0$ for all $n$ and where $\left\{f_{n}\right\}$ is a sequence in the extended complex plane defined by

$$
f_{n}=S_{n}(0), \quad n=0,1,2, \ldots
$$


Here $\left\{S_{n}(w)\right\}$ is the sequence of linear fractional transformations

$$
S_{0}(w)=s_{0}(w) ; \quad S_{n}(w)=S_{n-1}\left(s_{n}(w)\right), \quad n=1,2,3, \ldots,
$$

where

$$
s_{0}(w)=b_{0}+w ; \quad s_{n}(w)=\frac{a_{n}}{b_{n}+w}, \quad n=1,2,3, \ldots
$$

The numbers $a_{n}, b_{n}$ are called the elements of the continued fraction and $f_{n}$ is called the $n$th approximant. For convenience we usually denote a continued fraction $\left\langle\left\langle\left\{a_{n}\right\},\left\{f_{n}\right\}\right\rangle,\left\{f_{n}\right\}\right\rangle$ by one of the symbols

$$
b_{0}+\mathbf{K}\left(\frac{a_{n}}{b_{n}}\right), \quad b_{0}+\underset{n=1}{\mathbf{K}}\left(\frac{a_{n}}{b_{n}}\right) \text { or } b_{0}+\frac{a_{1}}{b_{1}}+\frac{a_{2}}{b_{2}}+\frac{a_{3}}{b_{3}}+\cdots
$$

A continued fraction is said to converge if its sequence of approximants $\left\{f_{n}\right\}$ converges to a point in the complex plane. When convergent, the continued fraction is said to have the value given by $\lim f_{n}$. The symbols (1.10) may be used to denote both the continued fraction and its value. When $\left\{a_{n}\right\}$ and $\left\{b_{n}\right\}$ are infinite sequences, then $b_{0}+K\left(a_{n} / b_{n}\right)$ is called an infinite (or nonterminating) continued fraction. It is called a finite (or terminating) continued fraction if $\left\{a_{n}\right\}$ and $\left\{b_{n}\right\}$ have only a finite number of terms $a_{1}, a_{2}, \ldots, a_{m}$ and $b_{0}, b_{1}, b_{2}, \ldots, b_{m}$. A continued fraction is assumed to be nonterminating unless otherwise stated.

Corresponding to each continued fraction $b_{0}+K\left(a_{n} / b_{n}\right)$, there are sequences of complex numbers $\left\{A_{n}\right\},\left\{B_{n}\right\}$ defined by the second order linear difference equations

$$
\begin{aligned}
A_{-1} & =1, \quad A_{0}=b_{0}, & B_{-1}=0, & B_{0}=1, \\
A_{n} & =b_{n} A_{n-1}+a_{n} A_{n-2}, & n & =1,2,3, \ldots, \\
B_{n} & =b_{n} B_{n-1}+a_{n} B_{n-2}, & n & =1,2,3, \ldots .
\end{aligned}
$$

The numbers $A_{n}$ and $B_{n}$ are called the $n$th numerator and denominator of $b_{0}+$ $\mathrm{K}\left(a_{n} / b_{n}\right)$, respectively. Some basic properties of the $A_{n}$ and $B_{n}$ are the following:

$$
\begin{gathered}
S_{n}(w)=\frac{A_{n}+A_{n-1} w}{B_{n}+B_{n-1} w}, \quad A_{n} B_{n-1}-A_{n-1} B_{n} \neq 0, \quad n=0,1,2, \ldots, \\
f_{n}=S_{n}(0)=\frac{A_{n}}{B_{n}}, \quad n=0,1,2, \ldots, \\
A_{n} B_{n-1}-A_{n-1} B_{n}=(-1)^{n-1} \prod_{k=1}^{n} a_{k}, \quad n=1,2,3, \ldots
\end{gathered}
$$

Equation (1.14) is called the determinant formula. In the following sections we also deal with continued fractions $K\left(a_{n}(z) / b_{n}(z)\right)$ whose elements $a_{n}(z)$ and $b_{n}(z)$ are polynomials in the complex variable $z$ with complex coefficients. The definitions and elementary properties given above are easily extended to include this case.

2. Existence of general $T$-fractions corresponding to given fLs at 0 and $\infty$. The concept of correspondence of a continued fraction to a fLs plays an important role in the sequel. Hence it will now be defined. First we note that the set $\mathcal{E}$ of all 
formal Laurent series (fLs)

$$
L=\sum_{n=-r}^{\infty} \alpha_{n} z^{n}, \quad r \text { an integer, } \alpha_{n} \in \mathbf{C},
$$

with increasing powers of $z$, forms a field with respect to addition and multiplication defined in the manner suggested by (2.1). $L=0$ is the zero element of $\mathcal{L}$. If $f(z)$ is a function meromorphic at the origin (i.e. in an open disk containing the origin), then its Laurent expansion, which is convergent in a deleted neighborhood of the origin, will be denoted by $\Lambda_{0}(f)$. A continued fraction $K\left(a_{n}(z) / b_{n}(z)\right)$, where $a_{n}(z)$ and $b_{n}(z)$ are polynomials in $z$ for all $n \geqslant 1$, will be said to correspond to a fLs, $L \in \mathcal{L}$, at $z=0$ if there exists a sequence $\left\{k_{n}\right\}$ of positive integers, with $\lim _{n \rightarrow \infty} k_{n}=\infty$, such that

$$
\Lambda_{0}\left(\frac{A_{n}(z)}{B_{n}(z)}\right)-L=g_{n} z^{k_{n}}+g_{n+1} z^{k_{n}+1}+\ldots
$$

Here $A_{n}(z)$ and $B_{n}(z)$ are the $n$th numerator and denominator of $\mathrm{K}\left(a_{n}(z) / b_{n}(z)\right)$, respectively. Both $A_{n}(z)$ and $B_{n}(z)$ are polynomials in $z$ and hence the $n$th approximant $A_{n}(z) / B_{n}(z)$ is a rational function of $z$.

Similarly the set of fLs

$$
L^{*}=\sum_{n=-s}^{\infty} \alpha_{-n}^{*} z^{-n}, \quad s \text { an integer, } \alpha_{-n}^{*} \in \mathbf{C},
$$

with decreasing powers of $z$, forms a field $\mathcal{L} *$. We denote by $\Lambda_{\infty}(f)$ the Laurent expansion at $z=\infty$ of a function $f(z)$ meromorphic at $z=\infty$. Then we say that a continued fraction $K\left(a_{n}(z) / b_{n}(z)\right)$, where $a_{n}(z)$ and $b_{n}(z)$ are polynomials in $z$, corresponds at $z=\infty$ to a fLs, $L^{*} \in \mathcal{L}^{*}$, if

$$
\Lambda_{\infty}\left(\frac{A_{n}(z)}{B_{n}(z)}\right)-L^{*}=h_{n} z^{-m_{n}}+h_{n+1} z^{-m_{n}-1}+\ldots,
$$

where $\left\{m_{n}\right\}$ is a sequence of positive integers with $\lim _{n \rightarrow \infty} m_{n}=\infty$.

By the order of correspondence we mean the two sequences $\left\{k_{n}\right\}$ and $\left\{m_{n}\right\}$, respectively.

In 1948, Thron [15] introduced continued fractions (1.4) with $F_{n}=1, n \geqslant 1$, as a means of expanding an arbitrary power series. Perron [12, pp. 173-175] generalized this to continued fractions equivalent to (1.4) with the restriction $F_{n} \neq 0, n \geqslant 1$, and observed that this continued fraction also corresponds to a fLs at $z=\infty$ (provided $G_{n} \neq 0, n \geqslant 1$ ). This phenomenon was further studied by Waadeland [17] and Jefferson [3].

Here one starts with an arbitrary fLs, $L \in \mathcal{L}$, finds a continued fraction $K$ corresponding to $L$ at $z=0$ and then observes that $K$ corresponds to another fLs, $L^{*} \in \mathcal{L}^{*}$, at $z=\infty$. Schematically this can be written $L \rightarrow K \rightarrow L^{*}$. The structurally different question, namely, given $L$ and $L^{*}$ to determine conditions on their coefficients to insure that there exists a continued fraction (1.4) corresponding to $L$ at 0 and $L^{*}$ at $\infty$, was first considered by Murphy and McCabe [8], [9] and independently somewhat later by Jones and Thron [4], [6], [16]. It is this question which is now answered by the following theorem. A proof is included since it has 
not been completely proved in any of the above references. The approximants of (1.4) turn out to be in the two-point Padé table determined by $L$ and $L^{*}$. We restrict ourselves here to the case where $r=-1$ and $s=0$.

THEOREM 2.1. Let fLs

$$
L=\sum_{n=1}^{\infty} \alpha_{n} z^{n} \text { and } L^{*}=\sum_{n=0}^{\infty} \alpha_{-n}^{*} z^{-n}
$$

be given. There exists a general $T$-fraction (1.4) which with $F_{n} \neq 0$ and $G_{n} \neq 0$ for all $n>1$ corresponds to $L$ at $z=0$ and to $L^{*}$ at $z=\infty$ iff

$$
\Delta_{n} \neq 0 \text { and } \Phi_{n} \neq 0 \text { for all } n=0,1,2, \ldots
$$

Here

$$
\Delta_{0}=1 ; \quad \Delta_{n}=\left|\begin{array}{cccc}
\delta_{-(n-1)} & \delta_{-(n-2)} & \ldots & \delta_{0} \\
\delta_{-(n-2)} & \delta_{-(n-3)} & \ldots & \delta_{1} \\
\vdots & \vdots & & \vdots \\
\delta_{0} & \delta_{1} & \ldots & \delta_{n-1}
\end{array}\right|, \quad n=1,2,3, \ldots,
$$

and

$$
\Phi_{0}=1 ; \quad \Phi_{n+1}=\left|\begin{array}{cccc}
\delta_{-(n-1)} & \delta_{-(n-2)} & \cdots & \delta_{1} \\
\delta_{-(n-2)} & \delta_{-(n-3)} & \cdots & \delta_{2} \\
\vdots & \vdots & & \vdots \\
\delta_{1} & \delta_{2} & \cdots & \delta_{n+1}
\end{array}\right|
$$

The $\delta_{k}$ are defined by

$$
\delta_{k}=\alpha_{k}^{*}-\alpha_{k}
$$

where it is understood that $\alpha_{k}=0$ for $k \leqslant 0$ and $\alpha_{k}^{*}=0$ for $k>1$. The order of correspondence is $\{n+1\}$ at $z=0$ and $\{n\}$ at $z=\infty$. The $F_{n}$ and $G_{n}$ of (1.4) are given by

$$
\begin{gathered}
F_{1}=-\Phi_{1} ; \quad F_{n}=\frac{-\Delta_{n-2} \Phi_{n}}{\Delta_{n-1} \Phi_{n-1}}, \quad n=2,3,4, \ldots, \\
G_{n}=\frac{-\Delta_{n-1} \Phi_{n}}{\Delta_{n} \Phi_{n-1}}, \quad n=1,2,3, \ldots
\end{gathered}
$$

Proof. In our proof we shall use the equivalent form for the general $T$-fraction (1.4) given by

$$
\frac{z}{e_{1}+d_{1} z}+\frac{z}{e_{2}+d_{2} z}+\frac{z}{e_{3}+d_{3} z}+\cdots,
$$

where the $F_{n}, G_{n}$ and $e_{n}, d_{n}$ are related by

$$
F_{1}=\frac{1}{e_{1}} ; \quad F_{n}=\frac{1}{e_{n-1} e_{n}}, \quad n=2,3,4, \ldots,
$$




$$
G_{n}=\frac{d_{n}}{e_{n}}, \quad n=1,2,3, \ldots
$$

The $n$th approximants of (1.4) and (2.10) are identical. Letting $A_{n}(z)$ and $B_{n}(z)$ denote the $n$th numerator and denominator of (2.10), respectively, we obtain from (1.11) that $A_{n}(z)$ and $B_{n}(z)$ can be written in the forms

$$
\begin{gathered}
A_{1}(z)=z, \\
A_{n}(z)=a_{n, 1} z+a_{n, 2} z^{2}+\cdots+a_{n, n} z^{n}, \quad n=2,3,4, \ldots,
\end{gathered}
$$

where

$$
a_{n, 1}=e_{2} e_{3} \cdots e_{n}, \quad a_{n, n}=d_{2} d_{3} \cdots d_{n}
$$

and

$$
B_{n}(z)=b_{n, 0}+b_{n, 1} z+\cdots+b_{n, n} z^{n}, \quad n=1,2,3, \ldots,
$$

where

$$
b_{n, 0}=e_{1} e_{2} \cdots e_{n}, \quad b_{n, n}=d_{1} d_{2} \cdots d_{n} .
$$

We now assume that there exists a general $T$-fraction in the equivalent form (2.10) with $e_{n} \neq 0$ and $d_{n} \neq 0$ for all $n>1$ corresponding to $L$ at $z=0$ and to $L^{*}$ at $z=\infty$. By use of the determinant formula (1.14), we have

$$
\frac{A_{n+1}(z)}{B_{n+1}(z)}-\frac{A_{n}(z)}{B_{n}(z)}-\frac{(-1)^{n} z^{n+1}}{B_{n}(z) B_{n+1}(z)} \text {. }
$$

Expanding the right side in increasing powers of $z$, we obtain, with the help of (2.12), a fLs of the form

$$
\frac{A_{n+1}(z)}{B_{n+1}(z)}-\frac{A_{n}(z)}{B_{n}(z)}=\frac{(-1)^{n} z^{n+1}}{\left(e_{1} e_{2} \cdots e_{n}\right)^{2} e_{n+1}}+g_{n+2} z^{n+2}+g_{n+3} z^{n+3}+\ldots
$$

Similarly, expanding the right side of (2.13) in decreasing powers of $z$, we obtain with the help of (2.12) a fLs of the form

$$
\frac{A_{n+1}(z)}{B_{n+1}(z)}-\frac{A_{n}(z)}{B_{n}(z)}=\frac{(-1)^{n} z^{-n}}{\left(d_{1} d_{2} \cdots d_{n}\right)^{2} d_{n+1}}+h_{-(n+1)} z^{-(n+1)}+h_{-(n+2)} z^{-(n+2)}+\ldots
$$

It follows from (2.14) and (2.15) that the order of correspondence of (2.10) to $L$ is $\{n+1\}$ and to $L^{*}$ is $\{n\}$ as asserted. Thus we can expand the $n$th approximant $A_{n}(z) / B_{n}(z)$ in the form

$$
\Lambda_{0}\left(\frac{A_{n}(z)}{B_{n}(z)}\right)=\alpha_{1} z+\alpha_{2} z^{2}+\cdots+\alpha_{n} z^{n}+\gamma_{n+1}^{(n)} z^{n+1}+\gamma_{n+2}^{(n)} z^{n+2}+\ldots,
$$

where

$$
\alpha_{1}-\gamma_{1}^{(0)}=\frac{1}{e_{1}} ; \quad \alpha_{n+1}-\gamma_{n+1}^{(n)}=\frac{(-1)^{n}}{\left(e_{1} e_{2} \cdots e_{n}\right)^{2} e_{n+1}}, \quad n=1,2,3, \ldots,
$$


and in the form

$$
\begin{aligned}
\Lambda_{\infty}\left(\frac{A_{n}(z)}{B_{n}(z)}\right)= & \alpha_{0}^{*}+\alpha_{-1}^{*} z^{-1}+\cdots+\alpha_{-(n-1)}^{*} z^{-(n-1)} \\
& +\lambda_{-n}^{(n)} z^{-n}+\lambda_{-(n+1)}^{(n)} z^{-(n+1)}+\ldots
\end{aligned}
$$

where

$$
\alpha_{0}^{*}-\lambda_{0}^{(0)}=\frac{1}{d_{1}} ; \quad \alpha_{-n}^{*}-\lambda_{-n}^{(n)}=\frac{(-1)^{n}}{\left(d_{1} d_{2} \cdots d_{n}\right)^{2} d_{n+1}}, \quad n=1,2,3, \ldots
$$

It follows from (2.16) that $L B_{n}(z)-A_{n}(z)$ is a fLs of the form

$$
\begin{aligned}
& L B_{n}(z)-A_{n}(z) \\
& \quad=\left(e_{1} e_{2} \cdots e_{n}\right)\left(\alpha_{n+1}-\gamma_{n+1}^{(n)}\right) z^{n+1}+\mu_{n+2} z^{n+2}+\mu_{n+3} z^{n+3}+\ldots
\end{aligned}
$$

Equating coefficients of like powers of $z$ on both sides and using (2.12), we obtain the equations

$$
\begin{aligned}
& \alpha_{0} b_{n, 0}+\alpha_{-1} b_{n, 1}+\cdots+\alpha_{-n} b_{n, n}=a_{n, 0}=0, \\
& \left\{\begin{array}{l}
\alpha_{1} b_{n, 0}+\alpha_{0} b_{n, 1}+\cdots+\alpha_{-(n-1)} b_{n, n}=a_{n, 1}, \\
\alpha_{2} b_{n, 0}+\alpha_{1} b_{n, 1}+\cdots+\alpha_{-n} b_{n, n}=a_{n, 2} \\
\cdots \cdots \cdots+\alpha_{n-1} b_{n, 1}+\cdots+\alpha_{0} b_{n, n}=\alpha_{n, n} \\
\alpha_{n} b_{n, 0}+\alpha_{n+\cdots}
\end{array}\right.
\end{aligned}
$$

and

$$
\alpha_{n+1} b_{n, 0}+\alpha_{n} b_{n, 1}+\cdots+\alpha_{1} b_{n, n}=e_{1} e_{2} \cdots e_{n}\left(\alpha_{n+1}-\gamma_{n+1}^{(n)}\right) .
$$

We recall that $\alpha_{k}=0$ for $k \leqslant 0$. Similarly, $L^{*} B_{n}(z)-A_{n}(z)$ has a fLs of the form

$$
L^{*} B_{n}(z)-A_{n}(z)=\left(d_{1} d_{2} \cdots d_{n}\right)\left(\alpha_{-n}-\lambda_{-n}^{(n)}\right) z^{-n}+\nu_{-(n+1)} z^{-(n+1)}+\ldots,
$$

and equating coefficients of like powers of $z$ gives the equations

$$
\begin{gathered}
\alpha_{1}^{*} b_{n, n}+\alpha_{2}^{*} b_{n, n-1}+\cdots+\alpha_{n+1}^{*} b_{n, 0}=0, \\
\left\{\begin{array}{l}
\alpha_{0}^{*} b_{n, n}+\alpha_{1}^{*} b_{n, n-1}+\cdots+\alpha_{n}^{*} b_{n, 0}=a_{n, n} \\
\alpha_{-1}^{*} b_{n, n}+\alpha_{0}^{*} b_{n, n-1}+\cdots+\alpha_{n-1}^{*} b_{n, 0}=a_{n, n-1} \\
\cdots \cdots \cdots \cdots \cdots \cdots \cdots \cdots \cdots \cdots \cdots \cdots \cdots \cdots \cdots \cdots \cdots \\
\alpha_{-(n-1)}^{*} b_{n, n}+\alpha_{-(n-2)}^{*} b_{n, n-1}+\cdots \cdots+\alpha_{1}^{*} b_{n, 0}=a_{n, 1}
\end{array}\right.
\end{gathered}
$$

and

$$
\alpha_{-n}^{*} b_{n, n}+\alpha_{-(n-1)}^{*} b_{n, n-1}+\cdots+\alpha_{0}^{*} b_{n, 0}=d_{1} d_{2} \cdots d_{n}\left(\alpha_{-n}^{*}-\lambda_{-n}^{(n)}\right) .
$$

Again we recall that $\alpha_{k}^{*}=0$ if $k>1$. By subtracting corresponding equations in (2.18) from (2.19), so as to eliminate $a_{n, 1}, a_{n, 2}, \ldots, a_{n, n}$, we obtain (by use of (2.8)) the equations

$$
\delta_{-n} b_{n, n}+\delta_{-(n-1)} b_{n, n-1}+\cdots+\delta_{0} b_{n, 0}=d_{1} d_{2} \cdots d_{n}\left(\alpha_{-n}^{*}-\lambda_{-n}^{(n)}\right),
$$




$$
\left\{\begin{array}{l}
\delta_{-(n-1)} b_{n, n}+\delta_{-(n-2)} b_{n, n-1}+\cdots+\delta_{0} b_{n, 1}=-\delta_{1} b_{n, 0} \\
\delta_{-(n-2)} b_{n, n}+\delta_{-(n-3)} b_{n, n-1}+\cdots+\delta_{1} b_{n, 1}=-\delta_{2} b_{n, 0} \\
\cdots \cdots \cdots \cdots \cdots \cdots \cdots \cdots+\cdots \cdots+\delta_{n-1} b_{n, 1}=-\delta_{n} b_{n, 0}
\end{array}\right.
$$

and

$$
\delta_{1} b_{n, n}+\delta_{2} b_{n, n-1}+\cdots+\delta_{n+1} b_{n, 0}=-e_{1} e_{2} \cdots e_{n}\left(\alpha_{n+1}-\gamma_{n+1}^{(n)}\right) .
$$

The determinant of the system (2.20b) is $\Delta_{n}$. Therefore an application of Cramer's rule yields the equations

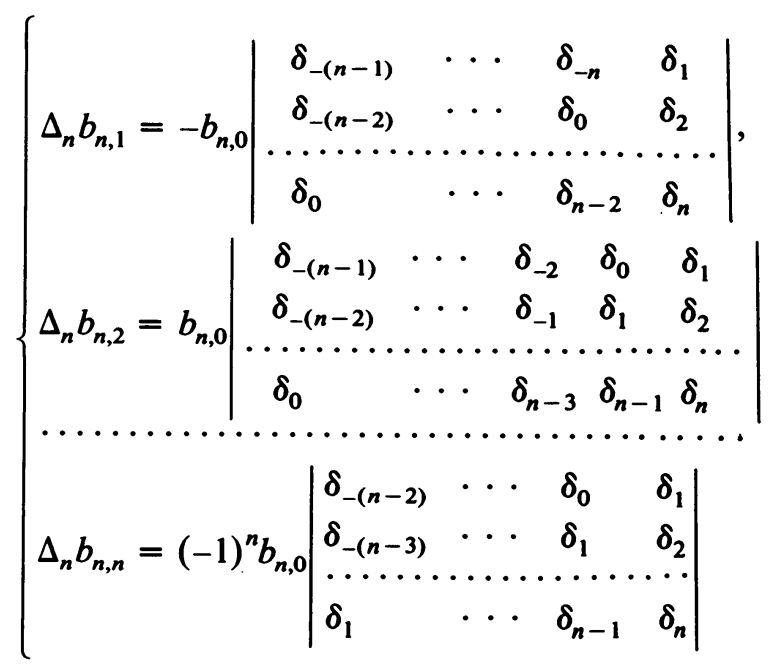

Now expanding the determinant $\Delta_{n+1}$ by cofactors along the first row and applying (2.21) gives

$$
\Delta_{n+1}=\frac{(-1)^{n} \Delta_{n}}{b_{n, 0}}\left(\delta_{-n} b_{n, n}+\delta_{-(n-1)} b_{n, n-1}+\cdots+\delta_{0} b_{n, 0}\right) .
$$

Combining this with $(2.12 \mathrm{e}),(2.20 \mathrm{a})$ and $(2.17 \mathrm{~b})$ gives

$$
\Delta_{1}=\frac{1}{d_{1}} ; \quad \Delta_{n+1}=\frac{\Delta_{n}}{\left(e_{1} e_{2} \cdots e_{n}\right)\left(d_{1} d_{2} \cdots d_{n} d_{n+1}\right)}, \quad n=1,2,3, \ldots
$$

It follows by induction from (2.22) that

$$
\Delta_{n} \neq 0, \quad n=1,2,3, \ldots
$$

Similarly, if $\Phi_{n+1}$ is expanded by cofactors along the last column, and (2.21) is applied, we obtain

$$
\Phi_{n+1}=\frac{\Delta_{n}}{b_{n, 0}}\left(\delta_{1} b_{n, n}+\delta_{2} b_{n, n-1}+\cdots+\delta_{n+1} b_{n, 0}\right) .
$$

Combining this with (2.20c), (2.12e) and (2.16b) gives

$$
\Phi_{1}=-\frac{1}{e_{1}} ; \quad \Phi_{n+1}=\frac{(-1)^{n-1} \Delta_{n}}{\left(e_{1} e_{2} \cdots e_{n}\right)^{2} e_{n+1}}, \quad n=1,2,3, \ldots
$$


It follows from this and (2.23) that

$$
\Phi_{n} \neq 0, \quad n=1,2,3, \ldots
$$

Using (2.11), (2.22) and (2.24), one can easily derive (2.9).

Conversely, we assume now that conditions (2.6) are satisfied. Then a general $T$-fraction (1.4) is defined by (2.9) and its coefficients satisfy $F_{n} \neq 0, G_{n} \neq 0$ for all $n=1,2,3, \ldots$ It follows from (2.14) and (2.15) that the continued fraction corresponds to a fLs

$$
\hat{L}=\sum_{n=1}^{\infty} \hat{\alpha}_{n} z^{n}
$$

at $z=0$ and to a fLs

$$
\hat{L}^{*}=\sum_{n=0}^{\infty} \hat{\alpha}_{-n}^{*} z^{-n}
$$

at $z=\infty$. Now the procedure used in the first part of the proof to define the coefficients $F_{n}$ and $G_{n}$ in terms of the $\alpha_{n}$ and $\alpha_{n}^{*}$ can be applied to the $\hat{\alpha}_{n}$ and $\hat{\alpha}_{n}^{*}$ and this will yield the same $F_{n}$ and $G_{n}$. It is readily shown that sequences $\left\{F_{n}\right\}$, $\left\{G_{n}\right\}$ uniquely determine sequences $\left\{\alpha_{n}\right\},\left\{\alpha_{n}^{*}\right\}$ by means of the relations (2.9). Thus we conclude that $\alpha_{n}=\hat{\alpha}_{n}$ and $\alpha_{n}^{*}=\hat{\alpha}_{n}^{*}$ for all $n$. This completes the proof.

It follows from (2.12) that, for the general $T$-fraction considered in Theorem 2.1, the $n$th numerator $A_{n}(z)$ and denominator $B_{n}(z)$ are polynomials in $z$ of degree $n$. Thus the $n$th approximant $A_{n}(z) / B_{n}(z)$ is the $(n, n)$ two-point Padé approximant determined by the pair of fLs $L, L^{*}$.

3. Positive $T$-fractions and their integral representations. General $T$-fractions (1.4) for which all $F_{n}$ and $G_{n}$ are positive are of particular interest because they have integral representations. We begin by characterizing double sequences $C$ for which there exists a positive $T$-fraction corresponding to $L_{0}(C)$ at $z=0$ and to $L_{\infty}(C)$ at $z=\infty$.

THEOREM 3.1. Let $C=\left\{c_{n}\right\}_{-\infty}^{\infty}$ be a double sequence of real numbers and let $L_{0}(C)$ and $L_{\infty}(C)$ be defined by (1.1). There exists a positive T-fraction

$$
\frac{F_{1} z}{1+G_{1} z}+\frac{F_{2} z}{1+G_{2} z}+\cdots, \quad F_{n}>0, \quad G_{n}>0, \quad n=1,2,3, \ldots,
$$

corresponding to $L_{0}(C)$ at $z=0$ and to $L_{\infty}(C)$ at $z=\infty$ iff

$$
\begin{gathered}
H_{n+1}^{(-n)}(C)>0, \quad n=0,1,2, \ldots, \\
H_{2 n}^{(-2 n)}(C)>0, \quad H_{2 n-1}^{(-(2 n-1))}(C)<0, \quad n=1,2,3, \ldots .
\end{gathered}
$$

Proof. We apply Theorem 2.1 with $\alpha_{n}=-c_{-n}$ for $n \geqslant 1, \alpha_{-n}^{*}=c_{n}$ for $n<0$. Then

$$
\delta_{n}=\alpha_{n}^{*}-\alpha_{n}=\left\{\begin{array}{l}
0-\left(-c_{-n}\right), \quad n=1,2,3, \ldots \\
c_{-n}-0, \quad n=0,-1,-2, \ldots
\end{array}\right.
$$


Thus $\delta_{n}=c_{-n}$ for all $n$. Hence by (2.7),

$$
\Delta_{n}=\left|\begin{array}{cccc}
c_{n-1} & c_{n-2} & \ldots & c_{0} \\
c_{n-2} & c_{n-3} & \ldots & c_{-1} \\
\vdots & \vdots & & \vdots \\
c_{0} & c_{-1} & \cdots & c_{-(n-1)}
\end{array}\right|, \quad n=1,2,3, \ldots
$$

and

$$
\Phi_{n+1}=\left|\begin{array}{cccc}
c_{n-1} & c_{n-2} & \cdots & c_{-1} \\
c_{n-2} & c_{n-3} & \cdots & c_{-2} \\
\vdots & \vdots & & \vdots \\
c_{-1} & c_{-2} & \cdots & c_{-(n+1)}
\end{array}\right|, \quad n=0,1,2, \ldots
$$

Elementary operations on these determinants then leads to

$$
\Delta_{n}=H_{n}^{(-(n-1))}(C), n \geqslant 1, \text { and } \Phi_{n+1}=H_{n+1}^{(-(n+1))}(C), n \geqslant 0 \text {. }
$$

From (2.9) we have that $F_{n}>0$ and $G_{n}>0$ holds for all $n$ iff

$$
\Delta_{n}>0, \quad n=1,2,3, \ldots,
$$

and

$$
\Phi_{2 n-1}<0, \quad \Phi_{2 n}>0, \quad n=1,2,3, \ldots
$$

Substituting (3.3) into (3.4) completes the proof of the theorem.

Existence of integral representations for continued fractions was proved for the case of $K\left(a_{n} z / 1\right), a_{n}>0$, by Stieltjes [13]. For $K\left(z /\left(1+d_{n} z\right)\right), d_{n}>0$, it was done by Jones and Thron [5]. Both proofs depend on an analysis of the zeros of the denominators $B_{n}(z)$ of the approximants $A_{n}(z) / B_{n}(z)$ of the continued fraction. For $K\left(z /\left(1+d_{n} z\right)\right), d_{n}>0$, this analysis was carried out in [15]. The results for positive $T$-fractions parallel those in [5]. We shall now sketch the steps involved in the proof.

Let $A_{n}(z)$ and $B_{n}(z)$ denote the $n$th numerator and denominator, respectively, of the positive $T$-fraction (3.1). Then by the difference equations (1.11), one can see that $A_{n}(z)$ and $B_{n}(z)$ are polynomials in $z$ of degree $n$ of the forms

$$
\begin{gathered}
A_{n}(z)=F_{1} z+\cdots+F_{1}\left(\prod_{k=2}^{n} G_{k}\right) z^{n}, \quad n \geqslant 2 ; \quad A_{1}(z)=F_{1} z, \\
B_{n}(z)=1+\cdots+\left(\prod_{k=1}^{n} G_{k}\right) z^{n}, \quad n=1,2,3, \ldots
\end{gathered}
$$

One now shows that the zeros $r_{m}^{(n)}, m=1,2, \ldots, n$, of $B_{n}(z)$ are distinct and negative. This is proved by the difference equations (1.11), observing that the zeros of $B_{n}(z)$ are separated by those of $B_{n-1}(z)$. Further, the zeros of $P_{n}(z)=A_{n}(z) / z$ separate the zeros of $B_{n}(z)$. This together with the fact that $P_{n}(0) / B_{n}(0)=F_{1}>0$ insures that $P_{n}(z) / B_{n}(z)$ has a partial fraction expansion of the form

$$
\frac{P_{n}(z)}{B_{n}(z)}=\sum_{m=1}^{n} \frac{p_{m}^{(n)}}{z-r_{m}^{(n)}},
$$


where all $p_{m}^{(n)}, m=1, \ldots, n$, are positive. Finally,

$$
\sum_{m=1}^{n} p_{m}^{(n)}=\lim _{z \rightarrow \infty} \frac{z P_{n}(z)}{B_{n}(z)}=\lim _{z \rightarrow \infty} \frac{A_{n}(z)}{B_{n}(z)}=\frac{F_{1}}{G_{1}}>0 .
$$

Now arrange the zeros $r_{k}^{(n)}$ of $B_{n}(z)$ as follows,

$$
0<-r_{1}^{(n)}<-r_{2}^{(n)}<\cdots<-r_{n}^{(n)},
$$

and define the step function $\psi_{n}(t)$ by

$$
\psi_{n}(t)=\left\{\begin{array}{l}
0, \text { for } 0 \leqslant t \leqslant-r_{1}^{(n)}, \\
\sum_{m=1}^{k} p_{m}^{(n)}, \text { for }-r_{k}^{(n)}<t \leqslant-r_{k+1}^{(n)}, 1<k \leqslant n, \\
\frac{F_{1}}{G_{1}}, \text { for }-r_{n}^{(n)}<t<\infty .
\end{array}\right.
$$

Then $\psi_{n}(t) \in \Psi$ for all $n \geqslant 1$ and the $n$th approximant of (1.3) can be written as a Stieltjes integral

$$
\frac{A_{n}(z)}{B_{n}(z)}=\int_{0}^{\infty} \frac{z d \psi_{n}(t)}{z+t}
$$

These results can be summarized as follows.

THEOREM 3.2. Let $A_{n}(z)$ and $B_{n}(z)$ denote the nth numerator and denominator of $a$ positive $T$-fraction (3.1). Then $A_{n}(z)$ and $B_{n}(z)$ are polynomials in $z$ of degree $n$ and the zeros $r_{m}^{(n)}$ of $B_{n}(z)$ are all distinct and negative and can be arranged in order such that

$$
0<-r_{1}^{(n)}<-r_{2}^{(n)}<\cdots<-r_{n}^{(n)} .
$$

The nth approximant $A_{n}(z) / B_{n}(z)$ has the partial fraction decomposition

$$
\frac{A_{n}(z)}{B_{n}(z)}=\sum_{m=1}^{n} \frac{z p_{m}^{(n)}}{z-r_{m}^{(n)}}, \quad n=1,2,3, \ldots,
$$

where $p_{m}^{(n)}>0$ for $m \geqslant 1$ and $\sum_{m=1}^{n} p_{m}^{(n)}=F_{1} / G_{1}$. If $\psi_{n}(t), 0<t<\infty$, is defined by (3.5), then $\psi_{n}(t) \in \Psi, n \geqslant 1$, and

$$
\frac{A_{n}(z)}{B_{n}(z)}=\int_{0}^{\infty} \frac{z d \psi_{n}(t)}{z+t}, \quad n=1,2,3, \ldots
$$

To proceed we shall make use of the following result, sometimes referred to as "Grommer's selection theorem."

Let $\left\{\psi_{n}(t)\right\}$ be a sequence of real-valued nondecreasing functions defined on $-\infty<t<\infty$, such that $c \leqslant \psi_{n}(t) \leqslant C$ for all $-\infty<t<\infty, n=1,2,3, \ldots$ Then there exists a real-valued nondecreasing function $\psi(t)$ defined on $-\infty<t<\infty$ such that $c<\psi(t) \leqslant C$ for all $-\infty<t<\infty$, and there exists a subsequence $\left\{n_{k}\right\}$ of positive integers such that $\lim _{k \rightarrow \infty} \psi_{m_{k}}(t)=\psi(t)$ for $-\infty<t<\infty$. Moreover, if $g(t)$ is a continuous complex-valued function of the real variable $t$ such that $\lim _{t \rightarrow \pm \infty} g(t)$ 
$=0$, then

$$
\lim _{k \rightarrow \infty} \int_{-\infty}^{\infty} g(t) d \psi_{n_{k}}(t)=\int_{-\infty}^{\infty} g(t) d \psi(t) .
$$

The preceding result is based on a lemma due to Helly and Bray. Proofs of the result can be found in [11, p. 240, Theorem 6], [12, pp. 207-211] and [18, p. 246, Theorem 64.2]. Before applying this result we study the convergence behavior of positive $T$-fractions.

THeOREM 3.3. Let $f_{n}(z)$ denote the nth approximant of a positive T-fraction (3.1). Then: (A) The sequences $\left\{f_{2 n-1}(z)\right\}$ and $\left\{f_{2 n}(z)\right\}$ converge uniformly on every compact subset of $R=[z:|\arg z|<\pi]$.

(B) Let $\left\{e_{n}\right\}$ and $\left\{d_{n}\right\}$ be sequences of positive numbers defined by

$$
\begin{gathered}
e_{1}=\frac{1}{F_{1}} ; \quad e_{2 n-1}=\prod_{k=1}^{n-1} F_{2 k} / \prod_{k=1}^{n} F_{2 k-1}, \quad n \geqslant 2 ; \\
e_{2 k}=\prod_{k=1}^{n} F_{2 k-1} / \prod_{k=1}^{n} F_{2 k}, \quad n \geqslant 1 ; \\
d_{n}=G_{n} e_{n}, \quad n=1,2,3, \ldots,
\end{gathered}
$$

(note that (3.6) is equivalent to (2.11)) so that the positive T-fraction (3.1) is equivalent to

$$
\frac{z}{e_{1}+d_{1} z}+\frac{z}{e_{2}+d_{2} z}+\frac{z}{e_{3}+d_{3} z}+\cdots, \quad e_{n}>0, \quad d_{n}>0 .
$$

The continued fraction (3.1) converges uniformly on every compact subset of $R$ iff

$$
\sum_{n=1}^{\infty} e_{n}=\infty \text { or } \sum_{n=1}^{\infty} d_{n}=\infty .
$$

Proof. It is readily shown that (3.1) is equivalent to (3.7) which in turn is equivalent to

$$
w\left(\frac{1}{d_{1} w+e_{1} / w}+\frac{1}{d_{2} w+e_{2} / w}+\frac{1}{d_{3} w+e_{3} / w}+\cdots\right),
$$

where $w^{2}=z$. We choose $w$ so that $|\arg w|<\pi / 2$ and hence $z \in R$. Since $d_{n}>0$ and $e_{n}>0$, we have $\left|\arg \left(d_{n} w+e_{n} / w\right)\right|<|\arg w|<\pi / 2$. By Van Vleck's criterion (see, for example, [12, p. 73]) we obtain the uniform convergence of $\left\{f_{2 n-1}(z)\right\}$ and $\left\{f_{2 n}(z)\right\}$ on compact subsets of $R$. The continued fraction converges iff

$$
\sum_{n=1}^{\infty}\left|d_{n} w+\frac{e_{n}}{w}\right|=\infty
$$

Clearly (3.9) holds iff (3.8) is satisfied. This completes the proof.

A continued fraction whose $n$th approximant is the $(2 n-1)$ th (or $2 n$ th) approximant $f_{2 n-1}(z)$ (or $f_{2 n}(z)$ ) is called the odd (or even) part of the positive $T$-fraction (3.1). Theorem 3.3(A) shows that both the odd and even parts of (3.1) converge uniformly on compact subsets of $R$. Combining Theorem 3.3 with the Grommer selection theorem (with $g(t)=z /(z+t)$ ), yields: 
THeOREM 3.4. Let a positive T-fraction (3.1) be given and let $f_{n}(z)$ denote its nth approximant. Then there exist two functions $\psi^{(\sigma)} \in \Psi, \sigma=0,1$, such that, for $z \in R$,

$$
\lim _{n \rightarrow \infty} f_{2 n+\sigma}(z)=\int_{0}^{\infty} \frac{z d \psi^{(\sigma)}(t)}{z+t}=G^{(\sigma)}(z), \quad \sigma=0,1 .
$$

Since the sequences $\left\{f_{2 n+\sigma}(z)\right\}$ converge uniformly on compact subsets of $R$, it follows that the functions $G^{(\sigma)}(z)$ are holomorphic for $z \in R, \sigma=0$, 1. Iff $\sum_{n=1}^{\infty} e_{n}=\infty$ or $\sum_{n=1}^{\infty} d_{n}=\infty$, then $\psi^{(0)}(t)=\psi^{(1)}(t)=\psi(t)$. Here the $e_{n}$ and $d_{n}$ are defined by (3.6).

We note that $\sigma$ in $\psi^{(\sigma)}(t)$ and $G^{(\sigma)}(z)$ denotes merely an index, not a derivative. In $\S 5$ we shall show that the $\psi^{(0)}$ are actually in $\Psi_{\infty}$.

4. Asymptotic expansions of positive $T$-fractions. In $\$ 2$ it was shown that a positive $T$-fraction (3.1) corresponds to a fLs $L_{0}(C)$ at $z=0$ and to a fLs $L_{\infty}(C)$ at $z=\infty$. We shall now show (in several steps) that the functions $G^{(\boldsymbol{\sigma})}(z)$, to which the odd and even parts of (3.1) converge in $R$, have $L_{0}(C)$ and $L_{\infty}(C)$ as asymptotic expansions.

THEOREM 4.1. Let a double sequence $C=\left\{c_{n}\right\}_{-\infty}^{\infty}$ satisfying (3.2) be given. Let (3.1) be the positive $T$-fraction that corresponds to $L_{0}(C)$ at $z=0$ and to $L_{\infty}(C)$ at $z=\infty$. Let $f_{n}(z)$ denote the nth approximant of $(3.1)$ and let $\psi^{(0)}(t)$ and $\psi^{(1)}(t)$ denote functions in $\Psi$ (whose existence is asserted by Theorem 3.4) such that

$$
\lim _{n \rightarrow \infty} f_{2 n+\sigma}(z)=\int_{0}^{\infty} \frac{z d \psi^{(\sigma)}(t)}{z+t}, \quad z \in R, \quad \sigma=0,1 .
$$

Then

$$
c_{k}=\int_{0}^{\infty}(-t)^{k} d \psi^{(\sigma)}(t), \quad k=0, \pm 1, \pm 2, \ldots
$$

Proof. The fact that the positive $T$-fraction (1.3) corresponds to $L_{0}(C)$ at $z=0$ implies that, for $n=1,2,3, \ldots$,

$$
f_{n}(z)=\sum_{m=1}^{n}-c_{-m} z^{m}+z^{n+1} h_{n}(z),
$$

where $h_{n}(z)$ is holomorphic in some circular disk about the origin. Next in terms of the step functions $\psi_{n}(t)$ of (3.5) (see Theorem 3.2), we have, for all $z \in R$,

$$
\begin{aligned}
f_{n}(z) & =\int_{0}^{\infty} \frac{z d \psi_{n}(t)}{z+t} \\
& =\int_{0}^{\infty}\left(\frac{z}{t}-\frac{z^{2}}{t^{2}}+\cdots+(-1)^{k-1} \frac{z^{k}}{t^{k}}-\frac{(-z)^{k+1}}{t^{k}(z+t)}\right) d \psi_{n}(t) \\
& =\sum_{m=1}^{k}-z^{m} \int_{0}^{\infty} \frac{d \psi_{n}(t)}{(-t)^{m}}+z^{k+1} \int_{0}^{\infty} \frac{d \psi_{n}(t)}{(-t)^{k}(z+t)}
\end{aligned}
$$

All integrals occurring above exist, since $\psi_{n}(t)=0$ for $0<t<-r_{1}^{(n)}$ and $\psi_{n}(t)=$ $F_{1} / G_{1}$ for $-r_{n}^{(n)} \leqslant t<\infty$, so that they are all proper Stieltjes integrals.

Subtracting the two expressions (4.1) and (4.2) with $k=n$ obtained for $f_{n}(z)$ and 
dividing by $z$, we arrive at

$$
\begin{aligned}
\int_{0}^{\infty} \frac{d \psi_{n}(t)}{(-t)}-c_{-1}=z\left(\sum _ { m = 2 } ^ { n } \left(c_{-m}\right.\right. & \left.-\int_{0}^{\infty} \frac{d \psi_{n}(t)}{(-t)^{m}}\right) z^{m-2} \\
& \left.-\left(h_{n}(z)-\int_{0}^{\infty} \frac{d \psi_{n}(t)}{(-t)^{n}(z+t)}\right) z^{n-1}\right) .
\end{aligned}
$$

By letting $z \rightarrow 0$, one arrives at

$$
c_{-1}=\int_{0}^{\infty}(-t)^{-1} d \psi_{n}(t)
$$

Using (4.4) in (4.3) and repeating the process $n$ times, one obtains

$$
c_{-m}=\int_{0}^{\infty}(-t)^{-m} d \psi_{n}(t), \quad 1 \leqslant m \leqslant n .
$$

By expanding the $n$th approximant $f_{n}(z)$ and the integral in the last term in (4.2) about $\infty$, one gets in a similar manner

$$
c_{m}=\int_{0}^{\infty}(-t)^{m} d \psi_{n}(t), \quad 0 \leqslant m \leqslant n-1 .
$$

We next consider the integrals $\int_{0}^{\infty} t^{-m} d \psi^{(\sigma)}(t)$. Let $0<\delta<\mu<\infty$. Then $\int_{\delta}^{\mu} t^{-m} d \psi^{(\sigma)}(t)$ exists for $\sigma=0,1$ and $m$ a positive integer. Moreover, for an arbitrary $\varepsilon>0$, one can find $n_{\varepsilon}^{(\sigma)}$ such that

$$
\int_{\delta}^{\mu} \frac{d \psi^{(\sigma)}(t)}{t^{m}}<\int_{\delta}^{\mu} \frac{d \psi_{2 n+\sigma}(t)}{t^{m}}+\varepsilon \quad \text { for } n>n_{\varepsilon}^{(\sigma)} .
$$

Hence

$$
\int_{\delta}^{\mu} \frac{d \psi^{(\sigma)}(t)}{t^{m}}<\int_{0}^{\infty} \frac{d \psi_{2 n+\sigma}(t)}{t^{m}}+\varepsilon \leqslant\left|c_{-m}\right|+\varepsilon, \quad \text { for } n>n_{\varepsilon}^{(o)} .
$$

Since $\varepsilon$ is an arbitrary positive number in the inequality above we conclude that

$$
\int_{\delta}^{\mu} \frac{d \psi^{(o)}(t)}{t^{m}} \leqslant\left|c_{-m}\right|, \quad m \geqslant 1
$$

From this both the existence of the integral as well as the inequality

$$
\int_{0}^{\infty} \frac{d \psi^{(\sigma)}(t)}{t^{m}} \leqslant\left|c_{-m}\right|, \quad m \geqslant 1,
$$

follow. An analogous argument, where however we need not worry about the integral near $t=0$, allows us to conclude that

$$
\int_{0}^{\infty} t^{m} d \psi^{(\sigma)}(t) \leqslant\left|c_{m}\right|, \quad m \geqslant 0
$$

also holds.

Combining (4.2) with (4.5) and $f_{n}(z)=\int_{0}^{\infty}(z /(z+t)) d \psi_{n}(t)$, we have, for $1<k$ $\leqslant 2 n+\sigma$,

$$
\int_{0}^{\infty} \frac{z d \psi_{2 n+\sigma}(t)}{z+t}=\sum_{m=1}^{k}-c_{-m} z^{m}-z^{k+1} \int_{0}^{\infty}\left(\frac{t}{z+t}\right) \frac{d \psi_{2 n+\sigma}(t)}{(-t)^{k+1}}
$$


Define

$$
g_{n, k}(z)=-\frac{1}{c_{-(k+1)}} \int_{0}^{\infty}\left(\frac{t}{z+t}\right) \frac{d \psi_{n}(t)}{(-t)^{k+1}} .
$$

Then, at least for real positive $z,\left|g_{n, k}(z)\right|<1$. In (4.9) take the limit as $n \rightarrow \infty$. Since all of the other limits exist, $\lim _{n \rightarrow \infty} g_{2 n+\sigma, k}(z)=g_{k}^{(\sigma)}(z)$ also exists and

$$
\left|g_{k}^{(\sigma)}(z)\right| \leqslant 1 \text { for } 0<z<\infty, k \geqslant 1 \text {. }
$$

Here the $\sigma$ in $g_{k}^{(\sigma)}(z)$ denotes an index and not a derivative. Thus on the one hand we have, for $0<z<\infty$,

$$
\int_{0}^{\infty} \frac{z d \psi^{(\sigma)}(t)}{z+t}=\sum_{m=1}^{k}-c_{-m} z^{m}-c_{-(k+1)} g_{k}^{(\sigma)}(z) z^{k+1}
$$

On the other hand,

$$
\int_{0}^{\infty} \frac{z d \psi^{(\sigma)}(t)}{z+t}=\sum_{m=1}^{k}-z^{m} \int_{0}^{\infty} \frac{d \psi^{(\sigma)}(t)}{(-t)^{m}}+z^{k+1} \int_{0}^{\infty} \frac{d \psi^{(\sigma)}(t)}{(-t)^{k}(z+t)}
$$

where all of the integrals exist since we know that

$$
\int_{0}^{\infty} \frac{z d \psi^{(\sigma)}(t)}{z+t} \text { and } \int_{0}^{\infty} \frac{d \psi^{(\sigma)}(t)}{(-t)^{m}}, \quad m \geqslant 1
$$

exist. Now set

$$
\int_{0}^{\infty} \frac{d \psi^{(\sigma)}(t)}{(-t)^{m}}=c_{-m}^{(\sigma)}
$$

and

$$
\frac{1}{c_{-(k+1)}} \int_{0}^{\infty} \frac{t d \psi^{(\sigma)}(t)}{(-t)^{k+1}(z+t)}=h_{k}^{(\sigma)}(z)
$$

Here, as before, the $\sigma$ in $h_{k}^{(\sigma)}(z)$ denotes an index and not a derivative. Then, by (4.7),

$$
\left|h_{k}^{(\sigma)}(z)\right| \leqslant\left|\frac{c_{-}^{(o)}(k+1)}{c_{-(k+1)}}\right| \leqslant 1,
$$

at least for positive $z$. From (4.12) we can write

$$
\int_{0}^{\infty} \frac{z d \psi^{(\sigma)}(t)}{z+t}=\sum_{m=1}^{k}-c_{-m}^{(\sigma)} z^{m}-c_{-(k+1)} h_{k}^{(\sigma)}(z) z^{k+1}
$$

Subtracting (4.14) from (4.11), transposing and dividing by $z$, one obtains

$$
c_{-1}^{(\sigma)}-c_{-1}=z\left(\sum_{m=2}^{k}\left(c_{-m}-c_{-m}^{(\sigma)}\right) z^{m-2}+c_{-(k+1)} z^{k-1}\left(g_{k}^{(\sigma)}(z)-h_{k}^{(\sigma)}(z)\right)\right) .
$$

Letting $z$ tend to 0 (through positive values) one obtains $c_{-1}=c_{-1}^{(\text {o) }}$. Repeating the process yields

$$
c_{-m}=\int_{0}^{\infty} \frac{d \psi^{(\sigma)}(t)}{(-t)^{m}} \quad \text { for all } m=1,2,3, \ldots
$$


Expanding $\int_{0}^{\infty}(z /(z+t)) d \psi^{(\sigma)}(t)$ about $z=\infty$ and using a similar argument to the one described above, one arrives at

$$
c_{m}=\int_{0}^{\infty}(-t)^{m} d \psi^{(\sigma)}(t), \quad m=0,1,2, \ldots
$$

This completes the proof.

Our next theorem is

THEOREM 4.2. Let a double sequence $C=\left\{c_{n}\right\}_{-\infty}^{\infty}$ be given and let $\psi \in \Psi$ be such that $c_{m}=\int_{0}^{\infty}(-t)^{m} d \psi(t), m=0, \pm 1, \pm 2, \ldots$ Then

$$
G(z)=\int_{0}^{\infty} \frac{z d \psi(t)}{z+t}
$$

is a holomorphic function of $z$, for $z \in R=[z:|\arg z|<\pi]$, and $L_{0}(C)$ and $L_{\infty}(C)$ are the asymptotic expansions of $G(z)$ with respect to $R_{\alpha}=[z:|\arg z|<\alpha], 0<\alpha<$ $\pi$, at $z=0$ and $z=\infty$, respectively.

Proof. That $G(z)$ exists and is holomorphic for $z \in R$ is well known (see, for example, [12, p. 186]). Next we have

$$
\begin{aligned}
G(z) & =\int_{0}^{\infty}\left(\frac{z}{t}-\frac{z^{2}}{t^{2}}+\cdots+(-1)^{n-1} \frac{z^{n}}{t^{n}}-\frac{(-z / t)^{n+1}}{1+z / t}\right) d \psi(t) \\
& =\sum_{m=1}^{n}-c_{-m} z^{m}+(-1)^{n}\left(\int_{0}^{\infty} \frac{d \psi(t)}{t^{n}(z+t)}\right) z^{n+1}
\end{aligned}
$$

Set

$$
h_{n}(z)=\frac{(-1)^{n}}{c_{-(n+1)}} \int_{0}^{\infty} \frac{d \psi(t)}{t^{n}(z+t)}=\frac{(-1)^{n}}{c_{-(n+1)}} \int_{0}^{\infty}\left(\frac{t}{z+t}\right) \frac{d \psi(t)}{t^{n+1}} .
$$

Note that, for $d>0$ and $0<|\alpha|<\pi / 2,\left|1+d e^{i \alpha}\right|>1$, and that, for $\pi / 2<|\alpha|<$ $\pi,\left|1+d e^{i \alpha}\right| \geqslant|\sin (\pi-\alpha)|=|\sin \alpha|$. Using this we have

$$
\left|\frac{t}{z+t}\right|=\frac{1}{|1+z / t|} \leqslant \frac{1}{|\sin \alpha|},
$$

for $0<t<\infty, z \in R_{\alpha}, 0<\alpha<\pi$. It follows that

$$
\left|h_{n}(z)\right| \leqslant \frac{1}{\sin \alpha},
$$

for $z \in R_{\alpha}, 0<\alpha<\pi, n=1,2,3, \ldots$ This establishes that $L_{0}(C)$ is the asymptotic expansion of $G(z)$ with respect to $R_{\alpha}$ at $z=0$. A very similar argument establishes that $L_{\infty}(C)$ is the asymptotic expansion of $G(z)$ with respect to $R_{\alpha}$ at $z=\infty$. This completes the proof.

An immediate consequence of the two preceding theorems is the following.

THEOREM 4.3. Let a double sequence $C=\left\{c_{n}\right\}_{-\infty}^{\infty}$, satisfying (3.2), be given. Let (3.1) be the positive $T$-fraction that corresponds to $L_{0}(C)$ at $z=0$ and to $L_{\infty}(C)$ at $z=\infty$. Finally, let $G_{1}(z)$ and $G_{0}(z)$ be the limits of the odd and even parts of (3.1), respectively. Then $L_{0}(C)$ and $L_{\infty}(C)$ are asymptotic expansions with respect to $R_{\alpha}$, $0<\alpha<\pi$, at $z=0$ and $z=\infty$, respectively, of both $G_{0}(z)$ and $G_{1}(z)$. 
5. Terminating positive $T$-fractions. Our main result is as follows.

TheOREM 5.1. Let $C=\left\{c_{n}\right\}_{-\infty}^{\infty}$ be a given double sequence. A terminating positive $T$-fraction

$$
\underset{m=1}{\mathbf{K}}\left(\frac{F_{m} z}{1+G_{m} z}\right)
$$

corresponds to $L_{0}(C)$ at $z=0$ and to $L_{\infty}(C)$ at $z=\infty$ iff there exists a rational function

$$
\begin{gathered}
F(z)=\sum_{m=1}^{N} \frac{p_{m} z}{z+t_{m}}, \quad p_{m}>0, \quad m=1,2, \ldots, N, \\
0<t_{1}<\cdots<t_{N},
\end{gathered}
$$

such that

$$
L_{0}(C)=\Lambda_{0}(F(z)), \quad L_{\infty}(C)=\Lambda_{\infty}(F(z))
$$

$(\Lambda$ is defined in $\$ 2$ ). Conditions (5.3) are equivalent to

$$
\begin{gathered}
c_{n}=\sum_{m=1}^{N}\left(-t_{m}\right)^{n} p_{m}, \quad n=0, \pm 1, \pm 2, \ldots, \\
p_{m}>0, \quad m=1,2, \ldots, N, \quad 0<t_{1}<t_{2}<\cdots<t_{N} .
\end{gathered}
$$

Proof. Clearly (5.3) is equivalent to (5.4a), where $F(z)$ is of the form (5.2). In $\$ 3$ it was shown that a terminating positive $T$-fraction (the $N$ th approximant of a nonterminating positive $T$-fraction) can be written in the form (5.2). It remains to prove that, if $F(z)$ is a rational function of the form (5.2) such that (5.3) holds, then there exists a terminating positive $T$-fraction (5.1) corresponding to $L_{0}(C)$ at $z=0$ and to $L_{\infty}(C)$ at $z=\infty$.

We begin with the assertion that if there exist two sequences of fLs,

$$
L_{\delta}^{(n)}=\sum_{m=1}^{\infty} \gamma_{m}^{(n)} z^{m} \text { and } L_{\infty}^{(n)}=\sum_{m=0}^{\infty} \beta_{m}^{(n)} z^{-m}
$$

and two sequences of positive numbers $\left\{F_{n}\right\},\left\{G_{n}\right\}$ such that $L_{0}(C)=L_{0}^{(1)}, L_{\infty}(C)$ $=L_{\infty}^{(1)}$ and

$$
L_{\delta}^{(n)}=\frac{F_{n} z}{1+G_{n} z+L_{0}^{(n+1)}}, \quad L_{\infty}^{(n)}=\frac{F_{n} z}{1+G_{n} z+L_{\infty}^{(n+1)}}, \quad n=1,2,3, \ldots,
$$

then the positive $T$-fraction

$$
\mathbf{K}\left(\frac{F_{n} z}{1+G_{n} z}\right)
$$

corresponds to $L_{0}(C)$ at $z=0$ and to $L_{\infty}(C)$ at $z=\infty$. The continued fraction (5.6) may be terminating. A proof of the correspondence to $L_{0}(C)$ can be made from the fact that

$$
L_{0}(C)=\frac{F_{1} z}{1+G_{1} z}+\cdots+\frac{F_{n} z}{1+G_{n} z+L_{0}^{(n+1)}}=S_{n}\left(L_{0}^{(n+1)}\right)
$$


where $S_{n}$ denotes the linear fractional transformation (1.9) for the continued fraction (5.6). Letting $A_{n}$ and $B_{n}$ denote the $n$th numerator and denominator, respectively, of (5.6), we obtain from (1.12) and (1.14) the relation

$$
L_{0}(C)-\frac{A_{n}}{B_{n}}=\frac{A_{n}+A_{n-1} L_{0}^{(n+1)}}{B_{n}+B_{n-1} L_{0}^{(n+1)}}-\frac{A_{n}}{B_{n}}=\frac{(-1)^{n} F_{1} F_{2} \cdots F_{n} z^{n} L_{0}^{(n+1)}}{B_{n}\left(B_{n}+B_{n-1} L_{0}^{(n+1)}\right)} .
$$

Expanding the right side of (5.7) in increasing powers of $z$, we see that the first nonzero term is of degree at least $(n+1)$. It follows that $(5.6)$ corresponds to $L_{0}(C)$ at $z=0$. A similar argument applies to $L_{\infty}(C)$.

In view of the preceding result it will suffice to determine a sequence $\left\{H_{n}(z)\right\}$ of functions holomorphic both at $z=0$ and at $z=\infty$ and satisfying $H_{1}(z)=F(z)$ and $H_{n}(0)=0$, and to determine sequences of positive numbers $\left\{F_{n}\right\},\left\{G_{n}\right\}$ such that

$$
H_{n}(z)=\frac{F_{n} z}{1+G_{n} z+H_{n+1}(z)}, \quad n=1,2,3, \ldots
$$

For then (5.5) will be satisfied with $L_{0}^{(n)}=\Lambda_{0}\left(H_{n}\right)$ and $L_{\infty}^{(n)}=\Lambda_{\infty}\left(H_{n}\right), n=$ $1,2,3, \ldots$ The process, if at all possible, is unique. If $H_{n+1}=0$ for some $n$, then the positive $T$-fraction (5.6) terminates.

We now show that every rational function $F(z)$ of the form (5.2) can be expressed by

$$
F(z)=\frac{z P_{N-1}(z)}{P_{N}(z)}
$$

where

$$
\begin{aligned}
& P_{0}(z)=\alpha_{0}, \quad P_{n}(z)=\alpha_{n} \prod_{m=1}^{n}\left(z+\zeta_{m}^{(n)}\right), \quad n=1,2,3, \ldots, \\
& \alpha_{n}>0 \quad \text { for } n=0,1,2, \ldots, \\
& 0<\zeta_{m}^{(n)}<\zeta_{m}^{(n-1)}<\zeta_{m+1}^{(n)}, \quad 1 \leqslant m \leqslant n, \quad n=2,3,4, \ldots .
\end{aligned}
$$

In particular $\alpha_{N}=1$ and $\zeta_{m}^{(N)}=t_{m}$ for $1 \leqslant m \leqslant N$. In fact, it is clear that $F(z)$ can be written in the form $F(z)=z Q(z) / P_{N}(z)$, where

$$
Q(z)=\sum_{m=1}^{N} p_{m} \prod_{\substack{\nu=1 \\ \nu \neq m}}^{N}\left(z+\zeta_{\nu}^{(N)}\right)
$$

Hence $Q(z)$ is a polynomial in $z$ of degree $N-1$. Moreover,

$$
Q\left(-\zeta_{r}^{(N)}\right)=\sum_{m=1}^{N} p_{m} \prod_{\substack{\nu=1 \\ \nu \neq m}}^{N}\left(-\zeta_{r}^{(N)}+\zeta_{\nu}^{(N)}\right)=p_{r} \prod_{\substack{\nu=1 \\ \nu \neq r}}^{N}\left(\zeta_{\nu}^{(N)}-\zeta_{r}^{(N)}\right) .
$$

It follows that $Q\left(-\zeta_{r}^{(N)}\right)=(-1)^{r+1} D_{r}$, where $D_{r}>0, r=1,2, \ldots, N$. From this we conclude that $Q(z)$ has at least $N-1$ distinct zeros on the negative real axis separating the zeros of $P_{N}(z)$. This accounts for all of the zeros of $Q(z)$. Finally $Q(z)>0$ for all $z \geqslant-\zeta_{1}^{(N)}$ and hence $Q(z)$ is of the form $P_{N-1}(z)$ as defined by (5.9). 
We shall complete the proof of the theorem by showing that a sequence $\left\{H_{n}\right\}$ can be found such that $H_{1}(z)=F(z)$ and each $H_{n}(z)$ has the form

$$
H_{n}(z)=\frac{z P_{N-n}(z)}{P_{N+1-n}(z)}, \quad 1 \leqslant n \leqslant N-1,
$$

where the $P_{n}(z)$ are polynomials of the form $(5.9 \mathrm{~b}, \mathrm{c}, \mathrm{d})$ and where the $H_{n}(z)$ satisfy equations (5.8). For if this is so, then

$$
F(z)=H_{1}(z)=\frac{F_{1} z}{1+G_{1} z}+\cdots+\frac{F_{N-1} z}{1+G_{N-1} z+z P_{0}(z) / P_{1}(z)} .
$$

Now $P_{0}(z)=\alpha_{0}>0$ and $P_{1}(z)=\alpha_{1} z+\alpha_{1} \zeta_{1}^{(1)}$, where $\alpha_{1}>0, \zeta_{1}^{(1)}>0$. Hence if we set $F_{N}=\alpha_{0} / \alpha_{1} \zeta_{1}^{(1)}$ and $G_{N}=1 / \zeta_{1}^{(1)}$, we have

$$
F(z)=\frac{F_{1} z}{1+G_{1} z}+\cdots+\frac{F_{N} z}{1+G_{N} z},
$$

where all $F_{n}, G_{n}, n=1,2, \ldots, N$, are positive.

From (5.8) and (5.10) we have, for $0<n \leqslant N-1$,

$$
\frac{z P_{N-1-n}(z)}{P_{N-n}(z)}=\frac{F_{n} z}{H_{n}(z)}-\left(1+G_{n} z\right)=\frac{F_{n} P_{N+1-n}(z)-\left(1+G_{n} z\right) P_{N-n}(z)}{P_{N-n}(z)},
$$

and hence

$$
z P_{N-1-n}(z)=F_{n} P_{N+1-n}(z)-\left(1+G_{n} z\right) P_{N-n}(z), \quad 0<n<N-1 .
$$

The requirement that $P_{N-1-n}(z)$ be a polynomial in $z$ of degree $N-1-n$ determines $F_{n}$ and $G_{n}$ completely

$$
\begin{aligned}
F_{n} & =\left(\alpha_{N-n} \prod_{\nu=1}^{N-n} \zeta_{\nu}^{(N-n)}\right) /\left(\alpha_{N+1-n} \prod_{\nu=1}^{N+1-n} \zeta_{\nu}^{(N+1-n)}\right), \\
G_{n} & =\left(\prod_{\nu=1}^{N-n} \zeta_{\nu}^{(N-n)}\right) /\left(\prod_{\nu=1}^{N+1-n} \zeta_{\nu}^{(N+1-n)}\right) .
\end{aligned}
$$

Clearly all $F_{n}$ and $G_{n}$ are positive. Next from (5.11) we have

$$
\begin{aligned}
-\zeta_{m}^{(N-m)} P_{N-1-n}\left(-\zeta_{m}^{(N-n)}\right) & =F_{n} P_{N+1-n}\left(-\zeta_{m}^{(N-n)}\right) \\
& =(-1)^{m} E_{m}^{(N-n-1)}, \quad m=1,2, \ldots, N-n,
\end{aligned}
$$

where $E_{m}^{(N-n-1)}>0$. This is the case since the zeros of $P_{N-n}(z)$ separate those of $P_{N+1-n}(z)$ and all zeros are simple. It follows that, for $n<N-1$, the $N-n-1$ zeros of $\boldsymbol{P}_{N-n-1}(z)$ are all distinct, negative and separate those of $\boldsymbol{P}_{N-n}(z)$.

Finally we shall show that $\alpha_{N-1-n}>0$ for $1<n<N-n$. Clearly $\alpha_{N}=1>0$. We assume that $\alpha_{N-n}>0$ and proceed by induction. By considering the coefficients of $z^{N+1-n}$ in (5.11), we obtain

$$
\alpha_{N-1-n}=\alpha_{N+1-n} F_{n}\left(\sum_{m=1}^{N+1-n} \zeta_{m}^{(N+1-n)}\right)-\alpha_{N-n}-\alpha_{N-n} G_{n}\left(\sum_{m=1}^{N-n} \zeta_{m}^{(N-n)}\right)
$$


From (5.12a) we obtain $\alpha_{N+1-n} F_{n}=\alpha_{N-n} G_{n}$ and hence (5.12b) becomes

$$
\alpha_{N-1-n}=\alpha_{N-n}\left(G_{n}\left(\sum_{m=1}^{N+1-n} \zeta_{m}^{(N+1-n)}-\sum_{m=1}^{N-n} \zeta_{m}^{(N-n)}\right)-1\right) .
$$

Thus $\alpha_{N-1-n}>0$, provided the second factor on the right side of the preceding equation is positive. In view of $(5.12 a)$ this is equivalent to the inequality

$$
\prod_{m=1}^{N+1-n} \zeta_{m}^{(N+1-n)}<\prod_{m=1}^{N-n} \zeta_{m}^{(N-n)}\left(\sum_{m=1}^{N+1-n} \zeta_{m}^{(N+1-n)}-\sum_{m=1}^{N-n} \zeta_{m}^{(N-n)}\right)
$$

For this purpose we define

$$
\begin{aligned}
a_{2 j-1} & =\zeta_{j}^{(N+1-n)}-\zeta_{j-1}^{(N-n)}, & j & =1,2, \ldots, N+1-n, \\
a_{2 j} & =\zeta_{j}^{(N-n)}-\zeta_{j-1}^{(N+1-n)}, & j & =1,2, \ldots, N-n .
\end{aligned}
$$

It follows from (5.9d) that each $a_{i}$ is positive. Since $\zeta_{j}^{(N+1-n)}=\sum_{m=1}^{2 j-1} a_{m}, j=$ $1,2, \ldots, N+1-n$, and $\zeta_{j}^{(N-n)}=\sum_{m=1}^{2 j} a_{m}, j=1,2, \ldots, N-n,(5.13)$ can be written in the equivalent form

$$
\prod_{k=1}^{N+1-n}\left(\sum_{m=1}^{2 k-1} a_{m}\right)<\prod_{k=1}^{N-n}\left(\sum_{m=1}^{2 k} a_{m}\right)\left(\sum_{j=1}^{N+1-n} a_{2 j-1}\right)
$$

Thus it will suffice to prove the inequality

$$
\prod_{k=1}^{q}\left(\sum_{m=1}^{2 k-1} a_{m}\right)<\prod_{k=1}^{q-1}\left(\sum_{m=1}^{2 k} a_{m}\right)\left(\sum_{j=1}^{q} a_{2 j-1}\right)
$$

for arbitrary positive numbers $a_{i}$ and for all $q=2,3,4, \ldots$ Our proof is by induction on $q$. For $q=2,(5.15)$ reduces to $a_{1}\left(a_{1}+a_{2}+a_{3}\right)<\left(a_{1}+a_{2}\right)\left(a_{1}+a_{3}\right)$, which is easily verified. Now assume that (5.15) holds for a given value of $q$. It remains to show that it also holds for $q$ replaced by $q+1$, that is,

$$
\prod_{k=1}^{q+1}\left(\sum_{m=1}^{2 k-1} a_{m}\right)<\prod_{k=1}^{q}\left(\sum_{m=1}^{2 k} a_{m}\right)\left(\sum_{j=1}^{q+1} a_{2 j-1}\right) \text {. }
$$

This can be written as

$$
\begin{aligned}
\prod_{k=1}^{q}\left(\sum_{m=1}^{2 k-1} a_{m}\right)\left(\sum_{m=1}^{2 q} a_{m}\right)+\prod_{k=1}^{q}\left(\sum_{m=1}^{2 k-1} a_{m}\right) a_{2 q+1} \\
\quad<\left(\sum_{j=1}^{q} a_{2 j-1}\right) \prod_{k=1}^{q}\left(\sum_{m=1}^{2 k} a_{m}\right)+a_{2 q+1} \prod_{k=1}^{q}\left(\sum_{m=1}^{2 k} a_{m}\right)
\end{aligned}
$$

or, equivalently,

$$
\begin{aligned}
0< & \left(\sum_{m=1}^{2 q} a_{m}\right)\left(\sum_{j=1}^{q} a_{2 j-1}\right) \prod_{k=1}^{q-1}\left(\sum_{m=1}^{2 k} a_{m}\right)-\prod_{k=1}^{q}\left(\sum_{m=1}^{2 k-1} a_{m}\right) \\
& +a_{2 q+1}\left(\prod_{k=1}^{q}\left(\sum_{m=1}^{2 k} a_{m}\right)-\prod_{k=1}^{q}\left(\sum_{m=1}^{2 k-1} a_{m}\right)\right) .
\end{aligned}
$$

That the first term on the right side of (5.17) is positive follows from the induction hypothesis (5.15). That the second term on the right side of (5.17) is positive can be 
verified directly. This completes the induction and our proof of Theorem 5.1.

For the modified moment problem we have established the following.

TheOREM 5.2. For a given double sequence $C=\left\{c_{n}\right\}_{-\infty}^{\infty}$, there exists a unique solution $\psi$ of the modified moment problem $c_{n}=\int_{0}^{\infty}(-t)^{n} d \psi(t), \psi \in \Psi_{F}, n=$ $0, \pm 1, \pm 2, \ldots$, iff there exists a rational function $F(z)$ of the form

$$
F(z)=\sum_{m=1}^{N} \frac{p_{m} z}{z+t_{m}}, \quad 0<t_{1}<\cdots<t_{N}, \quad p_{m}>0, \quad m=1,2, \ldots, N,
$$

such that $L_{0}(C)=\Lambda_{0}(F(z))$ and $L_{\infty}(C)=\Lambda_{\infty}(F(z))$.

We conclude this section by observing that if a nonterminating positive $T$-fraction is such that its odd (even) part converges to a function

$$
F(z)=\int_{0}^{\infty} \frac{z d \psi(t)}{z+t}, \quad \psi \in \Psi_{F}
$$

then $F(z)$ has the form

$$
F(z)=\sum_{m=1}^{N} \frac{z p_{m}}{z+t_{m}}, \quad 0<t_{1}<\cdots<t_{N}, \quad p_{m}>0, \quad m=1,2, \ldots, N,
$$

and hence the positive $T$-fraction corresponds to $\Lambda_{0}(F(z))$ at $z=0$ and to $\Lambda_{\infty}(F(z))$ at $z=\infty$. This is impossible by Theorem 5.1. Therefore the $\psi^{(\sigma)}$ in Theorem 3.4 satisfy $\psi^{(\sigma)} \in \Psi_{\infty}$.

6. The strong Stieljtes moment problem. We begin with a result giving a necessary condition for the strong Stieltjes moment problem to have a solution. The proof is modelled on the corresponding proof for the ordinary moment problem.

TheOREM 6.1. A function $\psi \in \Psi_{\infty}$ will generate a given double sequence $C=$ $\left\{c_{n}\right\}_{-\infty}^{\infty}$ of moments, by means of the relations

$$
c_{n}=\int_{0}^{\infty}(-t)^{n} d \psi(t), \quad n=0, \pm 1, \pm 2, \ldots
$$

only if the double sequence $C$ satisfies the condition (3.2), that is,

$$
\begin{gathered}
H_{n+1}^{(-n)}(C)>0, \quad n=0,1,2, \ldots, \\
H_{2 n}^{(-2 n)}(C)>0, \quad H_{2 n-1}^{(-(2 n-1))}(C)<0, \quad n=1,2,3, \ldots
\end{gathered}
$$

Proof. We consider the quadratic forms

$$
Q(s, n)=\sum_{i=-n}^{n} \sum_{j=-n}^{n}(-1)^{s} c_{i+j+s} u_{i} u_{j}
$$

If the strong Stieltjes moment problem has a solution $\psi \in \Psi_{\infty}$, then

$$
Q(s, n)=\int_{0}^{\infty}\left(t^{s} \sum_{i=-n}^{n} \sum_{j=-n}^{n}(-t)^{i} u_{i}(-t)^{j} u_{j}\right) d \psi(t)
$$

since, for finite sums, the order of summation and integration can be exchanged 
even though the integration is improper. Thus

$$
Q(s, n)=\int_{0}^{\infty} t^{s}\left(\sum_{i=-n}^{n}(-t)^{i} u_{i}\right)^{2} d \psi(t)
$$

Since $\psi$ is assumed to have infinitely many points of increase, it then follows that all $Q(s, n)$ are positive definite quadratic forms. That all $Q(s, n)$ are positive definite quadratic forms is equivalent to the condition that all determinants,

$$
\left|\begin{array}{llll}
(-1)^{s} c_{-2 n+s} & (-1)^{s} c_{-2 n+1+s} & \cdots & (-1)^{s} c_{-2 n+k+s} \\
(-1)^{s} c_{-2 n+1+s} & (-1)^{s} c_{-2 n+2+s} & \cdots & (-1)^{s} c_{-2 n+k+1+s} \\
\cdots & \cdots & \cdots & \cdots \\
(-1)^{s} c_{-2 n+k+s} & (-1)^{s} c_{-2 n+k+1+s} & \cdots & (-1)^{s} c_{-2 n+2 k+s}
\end{array}\right|,
$$

where $k=0,1, \ldots, 2 n$, be positive (see, for example, [18, p. 88]). For $s=0$ we get $H_{k+1}^{(-2 n)}(C)>0$ and, for $s=1,(-1)^{k+1} H_{k+1}^{(-(2 n-1))}(C)>0$. By choosing $k=2 n-1$ and $k=2 n$ for $s=0$ and $k=2 n-1$ and $k=2 n-2$ for $s=1$, one obtains the inequalities (6.2). This completes the proof.

Our second result concerns the uniqueness of solutions to the strong Stieltjes moment problem. We shall call two elements of $\Psi$ essentially equal if they differ in at most their points of discontinuity.

THEOREM 6.2. Let $C=\left\{c_{n}\right\}_{-\infty}^{\infty}$ be a double sequence satisfying conditions (6.2) and let the positive $T$-fraction

$$
\underset{n=1}{\infty}\left(\frac{F_{n} z}{1+G_{n} z}\right)
$$

corresponding to $L_{0}(C)$ at $z=0$ and to $L_{\infty}(C)$ at $z=\infty$ be convergent on compact subsets of $R=[z:|\arg z|<\pi]$ (see Theorem 3.3). Then the strong Stieltjes moment problem for the double sequence $C$ has exactly one solution $\psi$ and it is contained in $\Psi_{\infty}$. Here, essentially equal solutions are considered as equal.

Proof. That the moment problem has at least one solution followed from Theorem 4.1. Let $\psi$ be a solution. Let $A_{n}(z), B_{n}(z)$ denote the $n$th numerator and denominator of (6.3), respectively. Then, from Theorem 3.1 and Theorem 4.2,

$$
\frac{A_{n}(z)}{B_{n}(z)}=\sum_{m=0}^{n-1} c_{m} z^{-m}+c_{n} z^{-n} \varphi_{n}(z)
$$

and

$$
\int_{0}^{\infty} \frac{z d \psi(t)}{z+t}=\sum_{m=0}^{n-1} c_{m} z^{-m}+c_{n} z^{-n} \chi_{n}(z),
$$

where $\varphi_{n}(z)$ and $\chi_{n}(z)$ are bounded functions of $z$ at least for $0<M<z<\infty$. Hence,

$$
\begin{aligned}
\int_{0}^{\infty} \frac{z B_{n}(z) d \psi(t)}{z+t}-A_{n}(z) & =B_{n}(z)\left(\int_{0}^{\infty} \frac{z d \psi(t)}{z+t}-\frac{A_{n}(z)}{B_{n}(z)}\right) \\
& =B_{n}(z) c_{n} z^{-n}\left(\chi_{n}(z)-\varphi_{n}(z)\right)=\beta_{n}(z),
\end{aligned}
$$


where $\beta_{n}(z)$ is bounded as $z \rightarrow \infty$ through positive values. Next consider

$$
\int_{0}^{\infty} \frac{z\left(B_{n}(z)-B_{n}(-t)\right)}{z+t} d \psi(t)-A_{n}(z)=\beta_{n}(z)-\int_{0}^{\infty} \frac{z B_{n}(-t)}{z+t} d \psi(t) .
$$

The left-hand side of this equation is a polynomial in $z$ of degree at most $n$ with a zero constant term. This follows from the fact that $\left(B_{n}(z)-B_{n}(-t)\right) /(z+t)$ is a polynomial in $z$ of degree $n-1$. The right-hand side is a function which is bounded as $z \rightarrow \infty$ through positive values. It follows that

$$
A_{n}(z)=\int_{0}^{\infty} \frac{z\left(B_{n}(z)-B_{n}(-t)\right)}{z+t} d \psi(t)
$$

By considering the expansions of $A_{n}(z) / B_{n}(z)$ and $\int_{0}^{\infty}(z /(z+t)) d \psi(t)$ at $z=0$, one obtains

$$
\int_{0}^{\infty} \frac{z B_{n}(z) d \psi(t)}{z+t}-A_{n}(z)=\gamma_{n}(z) \cdot z^{n+1}
$$

where $\gamma_{n}(z)$ is a bounded function of $z$ for positive values of $z$ near $z=0$. Hence

$$
\int_{0}^{\infty} \frac{z\left(B_{n}(z)-B_{n}(-t)\right)}{z+t} d \psi(t)-A_{n}(z)=-z \int_{0}^{\infty} \frac{B_{n}(-t)}{z+t}+\gamma_{n}(z) z^{n+1} \text {. }
$$

Using (6.5) and (6.6), one gets

$$
\begin{aligned}
\int_{0}^{\infty} \frac{z B_{n}(-t)}{z+t} d \psi(t) & =\sum_{m=1}^{n}-z^{m} \int_{0}^{\infty} \frac{B_{n}(-t)}{(-t)^{m}} d \psi(t)+z^{n+1} \int_{0}^{\infty} \frac{B_{n}(-t) d \psi(t)}{(-t)^{n}(z+t)} \\
& =\gamma_{n}(z) z^{n+1}
\end{aligned}
$$

From this one concludes that $\int_{0}^{\infty}\left(B_{n}(-t) / t^{m}\right) d \psi(t)=0$ for $m=1,2, \ldots, n$. Hence, $\int_{0}^{\infty} P(t)\left(B_{n}(-t) / t^{n}\right) d \psi(t)=0$ for all polynomials $P(t)$ in $t$ of degree less than or equal to $n-1$. Hence, in particular,

$$
\int_{0}^{\infty}\left(\frac{B_{n}(-t)-B_{n}(z)}{z+t}\right) \frac{B_{n}(-t)}{(-t)^{n}} d \psi(t)=0 .
$$

It also follows from (6.7) that

$$
\int_{0}^{\infty} \frac{B_{n}(-t)}{(-t)^{n}(z+t)} d \psi(t)=z^{-n} \int_{0}^{\infty} \frac{B_{n}(-t) d \psi(t)}{z+t}
$$

Using (6.4) one arrives at

$$
\int_{0}^{\infty} \frac{\left(z B_{n}(z) / z^{n}\right)}{z+t} d \psi(t)-\frac{A_{n}(z)}{z^{n}}=\beta_{n}(z) \cdot z^{-n}
$$

Thus we obtain, from (6.10) and (6.11),

$$
\begin{array}{r}
\int_{0}^{\infty} \frac{z\left(\left(B_{n}(z) / z^{n}\right)-\left(B_{n}(-t) /(-t)^{n}\right)\right)}{z+t} d \psi(t)-\frac{A_{n}(z)}{z^{n}} \\
=z^{-n}\left(\beta_{n}(z)-\int_{0}^{\infty} \frac{z B_{n}(-t)}{z+t} d \psi(t)\right)
\end{array}
$$

The left-hand side of the above equation is a polynomial in $z^{-1}$ of degree at most $(n-1)$. The right-hand side is of order $z^{-n}$ as $z \rightarrow \infty$ through positive values. It 
follows that

$$
\frac{A_{n}(z)}{z^{n}}=\int_{0}^{\infty} \frac{z\left(\left(B_{n}(z) / z^{n}\right)-\left(B_{n}(-t) /(-t)^{n}\right)\right)}{z+t} d \psi(t) .
$$

From (6.9) and (6.12) we have

$$
\begin{aligned}
\frac{\left(B_{n}(z)\right)^{2}}{z^{n}} \int_{0}^{\infty} \frac{z d \psi(t)}{z+t}-\frac{A_{n}(z) B_{n}(z)}{z^{n}} & =z B_{n}(z) \int_{0}^{\infty} \frac{B_{n}(-t) d \psi(t)}{(-t)^{n}(z+t)} \\
& =z \int_{0}^{\infty} \frac{\left(B_{n}(-t)\right)^{2}}{(-t)^{n}(z+t)} d \psi(t)
\end{aligned}
$$

Dividing both the left side and the right side of the above equation by $\left(B_{n}(z)\right)^{2} / z^{n}$, one arrives at

$$
\int_{0}^{\infty} \frac{z d \psi(t)}{z+t}-\frac{A_{n}(z)}{B_{n}(z)}=\frac{z^{n+1}}{\left(B_{n}(z)\right)^{2}} \int_{0}^{\infty} \frac{\left(B_{n}(-t)\right)^{2} d \psi(t)}{(-t)^{n}(z+t)}
$$

For $z>0$ one then concludes that

$$
\frac{A_{2 n}(z)}{B_{2 n}(z)}<\int_{0}^{\infty} \frac{z d \psi(t)}{z+t}<\frac{A_{2 n-1}(z)}{B_{2 n-1}(z)}, \quad n=1,2,3, \ldots
$$

Since $\psi$ here is an arbitrary solution of the moment problem, it is essentially equal to the solution obtained from the convergent positive definite $T$-fraction (see, for example, [12, pp. 184-190] and [19, Chapter 8, §7]). From Theorem 5.2 we know that the solution obtained from a nonterminating positive $T$-fraction is in $\Psi_{\infty}$. This completes the proof of Theorem 6.2.

If the positive $T$-fraction (3.1) does not converge, then $\psi^{(0)}(t)$ and $\psi^{(1)}(t)$ are distinct functions (see Theorem 3.4) and thus for every choice of $\alpha>0, \beta>0$ the function

$$
\psi_{\alpha, \beta}(t)=\frac{\alpha \psi^{(0)}(t)+\beta \psi^{(1)}(t)}{\alpha+\beta}
$$

is also a solution of the strong Stieltjes moment problem. Hence in this case there are infinitely many solutions. We do not know whether the $\psi_{\alpha, \beta}$ exhaust the possible solutions. The following result has now been proved.

THEOREM 6.3. The strong Stieltjes moment problem for a double sequence $C=$ $\left\{c_{n}\right\}_{-\infty}^{\infty}$ has a solution iff $H_{n+1}^{(-n)}(C)>0, n=0,1,2, \ldots$, and $H_{2 n}^{(-2 n)}(C)>0$, $H_{2 n-1}^{(-(2 n-1))}(C)<0, n=1,2,3, \ldots$ The solution is unique iff the positive T-fraction (3.1), corresponding to $L_{0}(C)$ at $z=0$ and to $L_{\infty}(C)$ at $z=\infty$, converges (or equivalently iff $\Sigma e_{n}=\infty$ or $\Sigma d_{n}=\infty$, where $e_{n}$ and $d_{n}$ are defined as in Theorem 3.3).

\section{REFERENCES}

1. Z. Covindarajula, Recurrence relations for the inverse moments of the positive binomial variable, J. Amer. Statist. Assoc. 57 (1963), 468-473.

2. P. Henrici, Applied and computational complex analysis. II, Wiley, New York, 1977. 
3. T. Jefferson, Some additional properties of T-fractions, $\mathrm{Ph}$. D. Thesis, University of Colorado, Boulder, Colorado, 1969.

4. William B. Jones, Multiple point Padé tables, Padé and Rational Approximation (E. B. Saff and R. S. Varga, eds.), Academic Press, New York, 1977, pp. 163-171.

5. William B. Jones and W. J. Thron, Further properties of T-fractions, Math. Ann. 166 (1966), 106-118.

6. __ Two-point Padé tables and T-fractions, Bull. Amer. Math. Soc. 83 (1977), 388-390.

7. D. G. Kabe, Inverse moments of discrete distributions, Canad. J. Statist. 4 (1976), 133-142.

8. J. H. McCabe, A formal extension of the Padé-table to include two-point Padé quotients, J. Inst. Math. Appl. 15 (1975), 363-372.

9. J. H. McCabe and J. A. Murphy, Continued fractions which correspond to power series expansions at two points, J. Inst. Math. Appl. 17 (1976), 233-247.

10. W. Mendenhall and E. H. Lehman, Jr., An approximation of the negative moments of the positive binomial useful in life testing, Technometrics 2 (1960), 227-242.

11. I. P. Natanson, Theory of functions of a real variable. I, Ungar, New York, 1955.

12. O. Perron, Die Lehre von den Kettenbrüchen. II, Chelsea, New York, 1950.

13. T. J. Stieltjes, Recherches sur les fractions continues, Ann. Fac. Sci. Toulouse 8 (1894) J, 1-122; ibid. 9 (1894) A, 1-47.

14. D. L. Thomas, Reciprocal moments of linear combinations of exponential variates, J. Amer. Statist. Assoc. 71 (1976), 506-512.

15. W. J. Thron, Some properties of continued fractions $1+d_{0} z+K\left(z / 1+d_{n} z\right)$, Bull. Amer. Math. Soc. 54 (1948), 112-120.

16. , Two-point Padé tables, T-fractions and sequences of Schur, Padé and Rational Approximation (E. B. Saff and R. S. Varga, eds.), Academic Press, New York, 1977, pp. 215-226.

17. H. Waadeland, On $T$-fractions of certain functions with a first order pole at the point of infinity, Norske Vid. Selsk. Forh. (Trondheim) 40 (1967), 1-6.

18. H. S. Wall, Analytic theory of continued fractions, Van Nostrand, Princeton, N. J., 1948.

19. D. V. Widder, The Laplace transform, Princeton Univ. Press, Princeton, N. J., 1948.

Department of Mathematics, University of Colorado, Boulder, Colorado 80309 (Current address of W. B. Jones and W. J. Thron)

Department of Mathematics, University of Trondhem, Trondheim, Norway (Current address of H. Waadeland) 